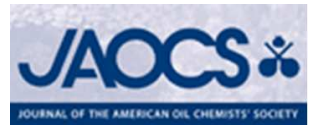

\title{
Rapid Determination of Olive Oil Chlorophylls and Carotenoids by Using Visible Spectroscopy
}

\begin{tabular}{|r|l|}
\hline Journal: & Journal of the American Oil Chemists Society \\
\hline Manuscript ID: & JAOCS-13-0514.R1 \\
\hline Manuscript Type: & Original Article \\
\hline Complete List of Authors: & $\begin{array}{l}\text { Cayuela, José; Instituto de la Grasa (CSIC), Fisiología y Tecnología de } \\
\text { Productos Vegetales } \\
\text { García, José; Instituto de la Grasa (CSIC), Fisiología y Tecnología de } \\
\text { Productos Vegetales; } \\
\text { Yousfi, Khaled; Instituto de la Grasa (CSIC), Fisiología y Tecnología de } \\
\text { Productos Vegetales } \\
\text { Martínez, María; Instituto de la Grasa, Fisiología y Tecnología de Productos } \\
\text { Vegetales }\end{array}$ \\
\hline Keywords: & $\begin{array}{l}\text { Fats and oils, Lipids, Antioxidants < Food and Feed Science / Nutrition and } \\
\text { Health, Oxidative Stability < Food and Feed Science / Nutrition and Health, } \\
\text { Spectroscopy < Lipid Chemistry / Lipid Analysis }\end{array}$ \\
\hline
\end{tabular}


1 Rapid Determination of Olive Oil Chlorophylls and Carotenoids by Using

*José A. Cayuela, Khaled Yousfi, M. Carmen Martínez, and José M. García

Instituto de la Grasa-CSIC, Avda. Padre García Tejero, 4, 41012, Sevilla

*Email: jacayuela@ig.csic.es; Telephone: +34 954611550

The determination of total chlorophylls and total carotenoids in olive oil by using visible spectroscopy (VIS) is reported. The technique proposed is rapid and non-destructive and can be used in a multi-parameter form. Moreover, it is environmentally friendly. The proposed technique has been compared with the determination of these pigments by near infrared spectroscopy (NIRS) and VIS together NIRS. Several procedures for multivariate regression were tested. The reference methods used were the determination of the extinction coefficient $\mathrm{K}_{670}$ for total chlorophylls and $\mathrm{K}_{470}$ for total carotenoids. A total of 258 samples were tested. The optimization of the calibration for total chlorophylls has been set by using multiple linear regression (MLR) from the wavelengths 670-686 nm exclusively visible. Its satisfactory performance is proven from the model coefficients standard error of calibration SEC 2.63 and $\mathrm{R}^{2} 0.97$, and the residual predictive deviation (RPD) 5.76 from the external validation. For the total carotenoids the best VIS calibration was fit by using the window of $465-475 \mathrm{~nm}$ and partial least squares (PLS), which provided RPD 3.68. However, the model built using the entire spectrum VIS-NIRS available (350$2500 \mathrm{~nm}$ ) was slightly better for this last pigment, showing RPD 3.86. Hence, this study 
23 shows the proposed VIS technique can be advantageous for the determination of total

24 chlorophylls in olive oils while is also suitable for determining total carotenoids.

Keywords: carotenoids, chlorophylls, multivariate analysis, olive oil, visible spectroscopy.

\section{Abbreviations}

FCV, full cross internal validation; MLR, multiple linear regression; NIR, near infrared; NIRS, near infrared spectroscopy; $\mathrm{PC}_{\mathrm{s}}$, principal components; $\mathrm{PCR}$, principal component regression; PLS, partial least squares; RPD, residual predictive deviation; SEC, standard error of calibration; SEP, standard error of performance; SLR, single linear regression; VIS, visible spectroscopy; VIS/NIRS, visible and near infrared spectroscopy; VOO, virgin olive oil; UV, ultraviolet; UV/VIS/NIR, ultraviolet, visible and near infrared;

\section{Introduction}

Olive oil can be consumed as a fruit juice, called virgin olive oil (VOO), which characterizes it and differentiates it from other plant oils. This oil, one of the main components of the Mediterranean diet, is recognized as a protector against cardiovascular diseases and cancer, due to its fatty acid composition and its content in phenolic compounds [1]. World production of olive oil ranges next to $3 \times 10^{6}$ t per year, Spain being the largest producer, with an average production from the last three seasons higher to $1.4 \times 10^{6} \mathrm{t}[2]$. A large increase in the demand for high quality VOO during recent years can be attributed not only to its particular sensory properties, but also to its potential health benefits. 
Several research results have demonstrated that plant pigments play important roles in 44 health [3]. The potential health benefit of a diet rich in carotenoids has been highlighted in multiple studies where their role as antioxidants and as agents which prevent cardiovascular diseases and degenerative eye pathologies is reported [4-5]. The provitamin A value of the carotenoids is well known. Numerous studies have also shown the anticancer activity of $\beta$ carotene and other carotenoids [6]. In addition, it has been reported that chlorophyll concentrations encountered in chlorophyll-rich green vegetables can provide substantial cancer chemoprotection, and suggested that they do so by reducing carcinogen 51 bioavailability [7].

On the other hand, carotenoids together with polyphenols and tocopherols provide oxidative stability to olive oils [2]. An important role of chlorophylls and carotenoids in the oxidative activity of processed foodstuff, due to their antioxidant nature in the dark and 55 pro-oxidant activity in the light, has been shown [8].

Moreover, the color of olive oil is an important visual attribute which has a decisive influence on the acceptance of the product [9]. Chlorophyll and carotenoid pigments in 58 olive oil greatly contribute to their color, so they are commonly used for the determination of olive oil color according to several methods [10-13]. These methods, instrumental and 60 visual, for olive oil color analysis and its relation with chlorophylls and carotenoids, have 61 been reviewed [10]. However, none of the above methods has been tuned for the rapid and 62 accurate determination of chlorophylls or carotenoids totals in olive oils.

63 Studies on the pigment composition in products derived from olives are relatively few [3].

64 The first high performance liquid chromatographic studies on the pigment fractions of 
mono-varietal VOOs from some Spanish olive varieties were done by Mínguez et al. [14-

15] and Gandul et al. [16-17]. Also the separation of olive oil chlorophyll and carotenoid pigments by thin layer chromatography and spectrophotometric identification has been described [18].

Mínguez et al. [19] describes a simple way for the determination of overall level of carotenoids and chlorophylls in olive oils which improved analysis speed, which is a factor worth pointing out. However, it still requires the use of solvents and a considerable amount of time for data processing. Taking into account the specific extinction coefficients of these pigments, and the usual level of concentration in olive oils, it is usually necessary a previous dilution of samples in order to achieve a measurable absorbance value. However, another way of getting these measurable absorbance values could be the use of a shorter pathlength during absorbance measurements.

In the context of raising competition in the olive sector, technological innovation must play an important role by providing the necessary improvements in the sector to live up to the circumstances. On the other hand, the environmental protection concern should be taken into account. Among the different actors involved in the production and marketing of olive oil, the industry plays a key role. In the olive mill, instant olive oil characterization is required to separate the different products in different deposits, according to their characteristics, thus maintaining its quality, identity and traceability. These intensive controls of olive oil quality would be possible only with analytical systems able to provide immediately and continuously all necessary information. Using the techniques available up to date, this is not possible yet satisfactorily. The non-destructive techniques are an 
87 alternative which is expected to provide new solutions, requiring its development and 88 validation.

89 With these purposes, Sikorska et al. [20] reported the capability of the fluorescence techniques to monitor chlorophyll content in oil products. Otherwise, the interaction 91 between pigments and near-infrared radiation $(750-2500 \mathrm{~nm})$ could provide useful correlations for determining olive oil chlorophylls and carotenoids. In fact, near infrared 93 spectroscopy (NIRS) has been reported multiple times for plant pigment determination [2194 27], as well the suitability of NIRS to determine the main olive oil quality parameters, such 95 as acidity, peroxides, K270 and K232, has been shown repeatedly [28-32]. Monitoring 96 carotenoid and chlorophyll in virgin olive oil has been reported by visible-near infrared transmittance spectroscopy (VIS-NIRS) [26], although in this study the separate analysis of 98 the wavelengths that contribute to the predictive models of chlorophylls and carotenoids is very scarcely reported. Nevertheless, the use of the VIS spectrum only could be better than their use together with the NIR region for predictive model for these pigments. The literature on the use of visible spectroscopy for the determination of quality parameters of olive oil is very little, very recently being reported the potential of UV-Visible spectroscopy as fingerprint technique in combination with chemometrics for classification of Spanish extra virgin olive oils [33]. Thus, there are much interest on clarify the respective contributions of visible and NIR regions in predictive models for these olive oil's compounds, as the simplicity of the technique may have a significant impact in reducing the cost of the instrument used. 
In the other hand, regarding multivariate methods, when the factors are few in number, are not significantly redundant and have a well-understood relationship to the responses, then multiple linear regression (MLR) can be a good way to turn data into information. However, if any of these three conditions breaks down, MLR can be inefficient or inappropriate. Partial Least Squares (PLS) is a method for constructing predictive models when the factors are many and highly collinear [34]. Principal Component Regression PCR) is also a popular method intended to overcome the problem of multicollinearity which arises with spectral data [35]. The difference between PLS and PCR is usually quite small, but PLS [36] will usually give results comparable to PCR-results using fewer components [37].

For this purposeTo answer the above questions, here is reported a study onmethod for determination of chlorophylls total and carotenoids total in olive oil by using VIS multivariate models. The contribution of the spectral regions ultraviolet (UV, 350-400 nm), VIS (400-779 nm) and NIR (780-2500 nm) to predictive models of total chlorophylls and total carotenoids of olive oil have been studied. from the spectral regions ultraviolet (UV, $350-400 \mathrm{~nm})$, VIS $(400-779 \mathrm{~nm})$ and NIR $(780-2500 \mathrm{~nm})$ is reported. Chlorophylls total and carotenoids total in olive oil have been determined by using VIS multivariate models, which performance were better than that obtained from single wavelengths. $A$ short optical pathlength of $5 \mathrm{~mm}$ was used for the purpose of getting measurable absorbance values without olive oil dilution. The procedures Single Linear Regression (SLR), Multiple Linear Regression (MLR), Principal Component Regression (PCR) and Partial Least Squares (PLS) were tested. The technique proposed is truly rapid and non-destructive, it does not require the use of solvents or reagents, it is environmentally friendly and can be 


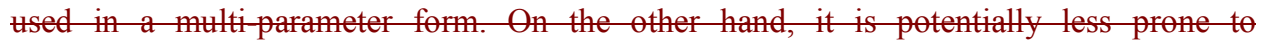

\section{Materials and methods}

\section{Virgin olive oils}

The development of predictive models was carried out based on spectra acquisition and reference analysis of VOO extracted in the Instituto de la Grasa (CSIC), from samples provided by a research project, taken in different olive groves of the provinces of Seville and Huelva. All the samples were extracted by a lab mill MC2 (Ingeniería y Sistemas, S.L., Spain). The VOO samples amounted to 258, and were composed of the varieties Picual, Arbequina and Manzanilla in equal quantities. The first two are quantitatively important cultivars in the production of olive oil in Spain. Manzanilla cv. is for table olives, but each campaign a small part of its production is used for olive oil extraction, with specific quality characteristics highly valued. The reason of including different olive cultivars in the study is to assure diversity in sample pigments composition. Olive oil samples were stored at $4^{\circ} \mathrm{C}$ until spectra acquisition.

Instrumentation

Spectral acquisition was carried out using an UV/VIS/NIR Labspec Pro (Analytical Spectral Devices Inc., Boulder). Labspec Pro is equipped with three detectors. The detector for the ultraviolet and visible range $(350-1000 \mathrm{~nm})$ is a fixed reflective holographic diode array. The wavelength range $1000-1800 \mathrm{~nm}$ is covered by a holographic fast scanner InGaAs detector cooled at $-25^{\circ} \mathrm{C}$. The same device before mentioned coupled with a high 
order blocking filter operates over an 1800-2500 nm interval. The instrument is equipped 153 with internal shutters and automatic offset correction, the scanning speed being $100 \mathrm{~ms}$. 154 The spectrometer can be used with a spectrophotometric cuvette accessory for 155 transmittance measurement, joined by fiber optic connectors to the light source spectrometer by one side of the accessory and to a detector on the opposite side.

The performance of the models built including the NIR spectrum together with the visible 159 is to be assessed. Hence, the temperature of the sample should be considered since it plays an important role on the NIR radiation it reflects and absorb [34ㅁ], and it constitutes a decisive factor in NIR spectroscopy. Therefore, the samples were taken from $4{ }^{\circ} \mathrm{C}$ storage and placed in a laboratory at approximately $25{ }^{\circ} \mathrm{C}$ for $18 \mathrm{~h}$ before processing. Prior to the recording of spectra, $125 \mathrm{~mL}$ containers of the samples were placed in a thermostated water bath fixed at $33^{\circ} \mathrm{C}$ for $30 \mathrm{~min}$, verifying temperature stability. The spectra acquisition was performed nondestructively in transmittance mode with each virgin olive oil sample, without further sample preparation, for two replicates of each sample. For the purpose of avoiding the use of cleaning solvents, the quartz cuvette was cleaned after each spectrum acquisition using oil excess from the next sample.

The whole spectrum comprised in the interval 350-2500 nm was acquired, each spectral variable corresponding to a $1 \mathrm{~nm}$ interval. The spectrometer was configured for continuous acquisition of 50 spectra, recorded their average as representative spectrum of the sample. Scans were performed in a Hellma quartz spectrophotometric cuvette with $5 \mathrm{~mm}$ path length. Indico Pro software (Analytical Spectral Devices Inc., Boulder) was used for this 
174 purpose. The acquisition process required for each sample was less than a minute, all steps 175 included.

\section{Reference analysis}

177 Clorophyll total and carotenoids total analyses in olive oils samples were determined 178 through the method described by Mínguez et al. [19]. Briefly, the chlorophyll and the 179 carotenoid fractions were evaluated from the absorption spectrum at $670 \mathrm{~nm}$ and $470 \mathrm{~nm}$ respectively, from the pigment extract in cyclohexane (Merck for spectroscopy, Darmstadt,

181 Germany), using the same solvent as blank reference. The absorption maximum at $670 \mathrm{~nm}$

182 in the spectrum of the total extract is due exclusively to the presence of the chlorophyll 183 fraction. As pheophytin "a" is the major component of this fraction, the group of 184 chlorophyll derivatives can be evaluated as if all were pheophytin "a", after calculating the coefficient of specific extinction in cyclohexane. The value in ethyl ether is $\varepsilon_{\mathrm{o}}=613 \mathrm{~nm}$ [19].

187 The spectrum of the carotenoid fraction takes the form of the dominant pigment, lutein. 188 Thus, it is possible to evaluate the yellow pigments in the total spectrum as if they are all lutein [19]. The maximum at $470 \mathrm{~nm}$ is chosen after obtaining the corresponding coefficient of extinction because it is a zone without interference from the pheophytin "a". This value

191 is reported $\varepsilon_{o}=2000[19]$.

192 For the procedure, $7.5 \mathrm{~g}$ oil were weighed exactly, dissolved in cyclohexane and taken to a 193 final volume of $25 \mathrm{~mL}$. Once the absorption spectrum was obtained, the chlorophyll total 
and carotenoids total fractions were deduced at $670 \mathrm{~nm}$ and $470 \mathrm{~nm}$ respectively, and

195 expressed as mg. $\mathrm{Kg}^{-1}$. All the samples were measured with two replicates.

Chemometry and calibration procedure

Prior to calibrations the transmittance data were transformed to absorbance and mean normalized. Savitzsky-Golay derivatives first and second were tested to see if they improve the performance of the models. The UV, VIS and NIR spectral regions were tested to prove the advantages these can provide, either using exclusively the NIR spectrum (1100-2500 $\underline{\mathrm{nm}}$ ), or by using the visible spectrum (400-1099 nm) together with UV (350-399 nm) and $\underline{\text { NIR. }}$

Partial Least Squares (PLS) was used for the selection of the specific spectral variables which should be included in the models. The procedure of selection consisted on performing several consecutive cycles of elimination of variables whose contribution to the model is null. It was made by selecting those variables whose spectral correlation coefficients with the parameter analyzed were closer to zero. This elimination was carried out by using the 'Mark with rectangle' option on the regression coefficients graph of The Unscrumbler. Variable selection ended in the last cycle which improved the statistical $\underline{\text { model } \mathrm{R}^{2} \text { and } \mathrm{R}^{2}} \underline{\text { CV. Model fitness was assessed by its standard error of calibration (SEC) }}$ and proximity between $\mathrm{R}^{2}$ and $\mathrm{R}^{2} \mathrm{Cv}$, independently from the model validation procedure below described.

The methods Principal Component Regression (PCR), Multiple Linear Regression (MLR) and Single Linear Regression (SLR) were tested. Single linear regression (SLR), multi linear regression (MLR), principal component regression (PCR), and partial least squares 
(PLS) [35] PLS, PCR, MLR and SLR models were obtained using The Unscrumbler (CAMO Software AS, Norway). The full cross internal validation (FCV) procedure was employed. The number of principal components $\left(\mathrm{PC}_{\mathrm{s}}\right)$ was fixed in the minimum to maximize the explanation of the parameter by the model. It was set for each calibration after tests parting from the number initial of $\mathrm{PC}_{\mathrm{s}}$ ten.

The UV, VIS and NIR spectral regions were tested to prove the advantages these can provides, either using exclusively the NIR - spectrum $(1100-2500 \mathrm{~nm})$, or by using the visible spectrum (400-1099 $\mathrm{nm})$ together with UV (350-399 $\mathrm{nm})$ and NIR. Additionally, the selection of the specific spectral variables which should be included in the models was conducted. The procedure of selection consisted on performing several consecutive cycles of elimination of variables whose contribution to the model is null. It was made by selecting those variables whose spectral correlation coefficients with the parameter analyzed were closer to zero. This elimination was carried out by using the 'Mark with rectangle' option on the regression coefficients graph of The Unscrumbler. Variable selection ended in the last cycle which improved the statistical model $\mathrm{R}^{z}$ and $\mathrm{R}^{z}{ }_{6}$. Model fitness was assessed by its standard error of calibration (SEC) and proximity between $R^{2}$ and $\mathrm{R}^{2}$, independently from the model validation procedure below described,

Model performance assessment

External validation exercises were conducted for both parameter analyzed. With this purpose, one fifth of the samples available was reserved and constituted a validation set. The calibration models previously built were used to predict the $\mathrm{K}_{670}$ and $\mathrm{K}_{470}$ extinction 
coefficients, corresponding to the chlorophyll total and carotenoids total contents 238 respectively, on the validation set.

239 The model performance was assessed mainly by the residual predictive deviation (RPD),

240 described as the ratio of the standard deviation $(\sigma)$ of the reference data from the validation

241 set to the standard error of performance (SEP) [3639].

242 Results and discussion

243 Olive oil visible spectrum

244 The assignment of the major visible absorption bands of olive oil has been described by 245 Moyano et al. [9]. Olive oil visible spectra characteristic of the Picual, Manzanilla and 246 Arbequina are shown in Figure 1. An initial peak appeared close to $420 \mathrm{~nm}$. This area 247 corresponds to the absorption by olive oil of wavelength dark blue colored light, which 248 could be due mainly to carotenoids, as well as to pheophytin a, pheophorbide a and 249 pyropheophytin a. A second peak near $460 \mathrm{~nm}$ was found, corresponding to the absorption 250 of the blue light, characteristic of carotenoids. Finally, a third peak was observed 251 approximately at $670 \mathrm{~nm}$, coinciding with the chlorophyll absorption.

253 Population characterization

254 The values from the parameters studied resulting from the reference analysis, 255 corresponding to the calibration and external validation sets, are included in Table 1. A 
the calibrations, as can be seen. The chlorophyll total and carotenoids total contents average from the calibration set analyzed were $17.23 \pm 15.83$ and $11.08 \pm 7.46 \mathrm{mg} \cdot \mathrm{Kg}^{-1}$, respectively.

\section{Spectral Variable Analysis and Chemometry}

The tests carried out using Savitzsky-Golay derivatives first and second didn't improve the fit of the models, whereas the absorbance data mean normalized provided the best outcomes. The visible window of 670-686 $\mathrm{nm}$ gave the best performance for predicting chlorophyll total, as result from the tests with different wavelength intervals, as is detailed below. On the contrary, the entire spectrum available $(350-2500 \mathrm{~nm})$ brought the best yields for carotenoids total. As is shown in Table 2, the PLS models built exclusively from NIR region provided $R^{2} 0.56$ and 0.62 for the predictive models of chlorophylls and carotenoids respectively, values which are too low for intend to use these models for analysis.

\section{Chlorophylls}

The tests to determinate the wavelengths with an optimal contribution to the total chlorophylls predictive calibration procedure of multivariate analysis-indicated the most appropriate suitable for total chlorophylls-was MLR $\left(\mathrm{Ch}_{1}\right)$ using the VIS window 670-686 $\mathrm{nm}$. The best procedure of multivariate analysis using this window was MLR $\left(\mathrm{Ch}_{1}\right)_{2}$ as proven from its statistics $\mathrm{R}^{2} 0.97$ and SEC 2.63, shown in Table 2. PLS $\left(\mathrm{Ch}_{2}\right)$ and PCR $\left(\mathrm{Ch}_{3}\right)$ using the same wavelengths showed lower performance, and very similar between both according their statistics, with $\mathrm{R}^{2} 0.96$ for both and SEC 3.20 and 3.22 (Table 2). Interestingly, both PLS and PCR, did provide R2 slightly lower only than MLR, but their SEC were $21.7 \%$ and $22.7 \%$ higher, hence they were worst. The calibration developed 
using VIS and NIR regions, by using the procedure previously described for selection of spectral variables $\left(\mathrm{Ch}_{4}\right)$, did not improve statistical indicators of calibration neither the predictive performance compared to $\mathrm{Ch}_{1}$, as it was proved by the data shown in Table 2 , with SEC 3.13. Particularly noteworthy is the predictive model obtained by SLR $\left(\mathrm{Ch}_{5}\right)$ with the only variable corresponding to a spectral wavelength of $670 \mathrm{~nm}$, the same used in the $\mathrm{K}_{670}$ reference, showed low efficacy with SEC 5.71. This fact emphasizes that using one only wavelength is not a better approach for determining chlorophylls by visible spectroscopy than using the multivariate method with the window $670-686 \mathrm{~nm}$. Likewise, the models built using MLR with the window restricted to $670-672 \mathrm{~nm}$ spectral variables were no better (data not shown). On the other hand, the calibration built exclusively using NIR $\left(\mathrm{Ch}_{6}\right)$ gave the worst performance among the methods assessed, as evidenced by their SEC 10.47 and $\mathrm{R}^{2} 0.56$ values (Table 2). These data reveal that chlorophyll content of the olive oil does not appear to be significant in the NIR spectrum. The good fit among prediction and measurement from the data used for the chlorophyll total calibration $\mathrm{Ch}_{1}$ using the VIS window 670-686 nm is shown in Figure 2.

The assessment of the models was mainly carried out by using them to determine the chlorophyll total content of a set of 54 samples, which was initially reserved for this purpose. The RPD of each multivariate procedure from the same validation set, proves the results referred above. The dispersion plot of this prediction is demonstrated in Figure 2 $\left(\mathrm{Vch}_{1}\right)$. As it's evidenced by the RPD value 5.76, shown in Table 2, the predictive model performance was very satisfactory. This ratio, the most widely used for determining the acceptability of a model, must reach the threshold of 3 for this purpose [36], which was fairly exceeded. 
VIS/NIR transmittance spectroscopy has been reported successful for on-line monitoring

carotenoid and chlorophyll pigments in virgin olive oil [26], where the separate analysis of the wavelengths contributing to the predictive models of chlorophylls and carotenoids was very scarce. The PLS model carried out in the present work including NIR together VIS regions $\left(\mathrm{Ch}_{4}\right)$, similarly to the work is reported above [26], provides RPD 4.05 which shows is valid for practical use and agrees with the study referred. However, its performance was clearly lower to the model built using wavelengths VIS only.

\section{Figure 2}

The spectral windows more suitable for build the models were slightly different among the different multivariate analysis methods (data not shown), that highlights the interaction between the variables and the model method. Hence, it reveals that most appropriate spectral variables to predict a certain parameter may depend on the nature of the predictive model.

\section{Carotenoids}

The tests to determinate the wavelengths with an optimal contribution to the total carotenoids predictive calibration revealed the most suitable was the VIS window 465-475 $\underline{\mathrm{nm}}$. The PLS $\left(\mathrm{Ca}_{1}\right)$ and PCR $\left(\mathrm{Ca}_{2}\right)$ models using thise visible wavelengths of $465-475 \mathrm{~nm}$ window developed using PLS $\left(\mathrm{Ca}_{1}\right)$ and PCR (Caz)-showed statistics SEC 2.28 and 2.30 and $\mathrm{R}^{2} 0.91$ and 0.90 , which are very similar, and RPD 3.68 from the external validations both (Table 2). These results demonstrate that using this window of the visible spectrum is suitable to determine total carotenoids. The calibration developed using MLR with the same wavelengths $465-475 \mathrm{~nm}\left(\mathrm{Ca}_{3}\right)$ showed statistics similar to those from PLS and PCR, 
although the SEP 2.11 and RPD 3.31 from the external validation were worst. This gospel agrees with the possibility of deleting factors, thus reducing dimensionality, what constitutes the major advantage of PCR regarding MLR. The possible advantage of PLS

over PCR is that it incorporates more information in the model-building phase [35], and

reflected actually on the $\mathrm{R}^{2}$ and SEC of the predictive models of total carotenoids built by PLS, slightly better than that of PCR.

Nevertheless, the best performance for predicting the total carotenoids of the olive oils was brought by the PLS model built from the entire spectrum available of 350-2500 nm (Ca4), being proven for the calibration SEC 1.74 and $R^{2} 0.95$ and RPD 3.86 from the external validation exercises, shown in Table 2 . As in the case of predicting total chlorophyll, this result agrees with the previously referred study [26], which reported as successful the online monitoring of the total carotenoids of virgin olive oil using VIS/NIRS.

Again we must emphasize that models constructed by SLR $\left(\mathrm{Ca}_{5}\right)$ from the single spectral variable $470 \mathrm{~nm}$ used in the reference method showed no better performance than using the wavelengths $465-475 \mathrm{~nm}$, that is demonstrated by the SEP 2.63 and RPD 2.66 from the external validation and by the calibration $\mathrm{R}^{2} 0.81$ and SEC 3.27, included in Table 2. As well, the PLS model developed from exclusively NIR region for predicting total carotenoids provided the worst outcome, with $\mathrm{R}^{2} 0.62$, value which is too low for intend to practical use. The good fit among predictions and measurements from the calibration for total carotenoids from the exclusively visible window of $465-475 \mathrm{~nm}$ is shown in Figure 3 $\left(\mathrm{Ca}_{1}\right)$ and the same from the external validation $\left(\mathrm{Vca}_{1}\right)$. The PLS model VIS/NIRS from

Formatted: Superscript 
350-2500 $\mathrm{nm}\left(\mathrm{Ca}_{4}\right)$ and the corresponding validation $\left(\mathrm{Vca}_{4}\right)$ are shown in Figure 4. As can 346 be seen, both models showed performance fairly similar.

Figure 3

\section{Conclusions}

Multivariate models set in this work from the wavelength intervals $670-686 \mathrm{~nm}$ for total chlorophylls and 465-475 $\mathrm{nm}$ for total carotenoids clearly improved those built by SLR from the single wavelengths used in the reference methods. None of the wavelengths from the spectrum NIR or UV regions assessed in this study provided any improvement in the performance of the multivariate model for total chlorophylls.

The spectral variables used in the models were very few, allowing the use of VIS standard spectrophotometers, therefore relatively inexpensive. The technique proposed is truly rapid and non-destructive, and easy to use also. Moreover, it is potentially less prone to experimental errors, because there is neither sample weighing nor volume adjustment. Another major advantage is the possibility of a multi-parametric determination of chlorophylls and carotenoids from a single measurement. Last but not least, it should be noted that the technique proposed is environmentally friendly, avoiding a considerable consumption of solvents or reagents, which aids in avoiding environmental costs. In summary, the proposed technique can be advantageous for the determination of total chlorophylls and carotenoids.

This paper has analyzed the respective contribution of UV, NIR and VIS spectrum in predictive models of chlorophylls total and carotenoids total. None of the wavelengths from 
the spectrum NIR or UV regions assessed in this study provided any improvement in the predictive models visible may have the advantage of having less influence on the spectrum of the temperature, both environmental as of the samples.

\section{Acknowledgements}

The models using the exclusively visible wavelengths of $465-475 \mathrm{~nm}$, either using PLS or PCR, were successful for measuring total carotenoids. The model VIS/NIRS for total earotenoids using the entire spectrum available showed performance slightly higher in the external validation exercise.

It should be pointed out that multivariate models set from the wavelength intervals used in this work both total chlorophylls and total carotenoids clearly improved those built by SLR from the single wavelengths used in the reference methods. The spectral variables used in the models were very few, which allows for the use of VIS standard spectrophotometers, therefore relatively inexpensive. Another major advantage is the possibility of a multiparametric determination of chlorophylls and carotenoids from a single measurement.-Both factors may contribute significantly to determining the features of the equipment and adapting them to reasonable economic parameters. Also, the ease in use of the technique has to be highlighted. Last but not least, it should be noted that the technique proposed is environmentally friendly, avoiding a considerable consumption of solvents, which aids in avoiding environmental costs.-In summary, the proposed technique can be advantageous for the determination of total chlorophylls and carotenoids. 
388 The authors are indebted to the Junta de Andalucía (project AGR-6456) for its financial 389 support, and gratefully acknowledge Antonio Montero, of the Instituto de Recursos 390 Naturales y Agrobiología (CSIC) for his technical assistance. Our thanks are also due to the 391 group Quality and Purity of Oils, of the Instituto de la Grasa (CSIC), for the use of the 392 chromatographic equipment.

\section{$393 \quad$ Literature cited}

1. Ruíz V, Muriana FJ, Villar J (1998) Virgin olive oil and cardiovascular diseases. Plasma lipid profile and lipid composition of human erythrocyte membrane. Grasas Aceites 49: 9-29

2. International Olive Oil Council (2013) World olive oil figures http://www.internationaloliveoil.org Accessed Jul 2013

3. Giuffrida D, Salvo F, Salvo A, Cossignani L, Dugo G (2011) Pigments profile in monovarietal virgin olive oils from various Italian olive varieties. Food Chem 124: $1119-1123$

4. Kritchevsky SB (1999) $\beta$-carotene, carotenoids and the prevention of coronary heart disease. J Nutr 129: 5-8

5. Landrum JT, Bone RA (2001) Lutein, zeaxanthin and the macular pigment. Arch Biochem Biophys 385: 28-40

6. Van Poppel G, Goldbohm RA (1995) Epidemiological evidence for $\beta$-carotene and cancer prevention. Am J Clin Nutr 62: 1493-1503 
7. McQuistan TJ, Simonich MT, Pratt MM, Pereira CB, Hendricks JD, Dashwood RH, Williams DE, Bailey GS (2012) Cancer chemoprevention by dietary chlorophylls: A 12,000-animal dose-dose matrix biomarker and tumor study. Food Chem Toxicol 50: $341-352$

8. Fakourelis N, Lee EC, Min DB (1987) Effects of chlorophylls and $\beta$-carotene on the oxidation stability of olive oil. J Food Sci 52: 234-235

9. Tous J, Romero A (1992) Caracterización del color de los aceites de oliva vírgenes de cultivares catalanes. Grasas Aceites 43: 347-351

10. Moyano MJ, Heredia FJ, Meléndez AJ (2010) The Color of olive oils: The pigments and their likely health benefits and visual and instrumental methods of analysis. Compr Rev Food Sci Saf 9: 278-291

11. Escolar D, Haro MR, Ayuso J (2002) An efficient method for a numerical description of virgin olive oil color with only two absorbance measurements. JAOCS 79: 769-774

12. Moyano MJ, Ayala F, Echávarri F, Alba J, Negueruela I, Herediad FJ (2001) Simplified measurement of virgin olive oil color by application of the characteristic vector method. JAOCS 78: 1221-1226

13. Moyano MJ, Meléndez AJ, Alba J, Heredia FJ (2008) A comprehensive study on the colour of virgin olive oils and its relationship with their chlorophylls and carotenoids indexes (I): CIEXYZ non-uniform colour space. Food Res Int 41: 505-512 
427 14. Minguez MI, Gandul B, Gallardo ML (1992) Rapid Method of quantification of 428 chlorophylls and carotenoids in virgin olive oil by high-performance liquid chromatography. J Agric Food Chem 40: 60-63

15. Mínguez MI, Gandul B, Garrido J, Gallardo ML (1990) Pigments present in virgin olive oil. JAOCS 67: 192-196

16. Gandul B, Mínguez I (1996) Chlorophyll and carotenoid composition in virgin olive oils from various Spanish olive varieties. J Sci Food Agric 72: 31-39

17. Gandul B, Gallardo L, Garrido J, Mínguez MI (1991) Control de pigmentos clorofílicos y carotenoides por HPLC en el aceite de oliva virgen. Grasas Aceites 42: $56-60$

18. Garrido J, Gandul B, Gallardo L, Minguez Ml (1990) Pigmentos clorofílicos y carotenoides responsables del color en el aceite de oliva virgen. Grasas Aceites 41: 404409

19. Mínguez MI, Rejano L, Gandul B, Higinio A, Garrido J (1991) Color-pigment correlation in virgin olive oil. JAOCS 68: 332-336

20. Sikorska E, Khmelinskii IV, Sikorski M, Caponio F, Bilancia MT, Pasqualone A, Gomes T (2008) Fluorescence spectroscopy in monitoring of extra virgin olive oil during storage. Int J Food Sci Tec 43: 52-61 
21. Sinelli N, Spinardi A, Egidio V, Mignani I, Casiraghi E (2008) Evaluation of quality and nutraceutical content of blueberries (Vaccinium corymbosum L.) by near and midinfrared spectroscopy. Postharvest Biol Tec 50: 31-36

22. Davei MW, Saeys W, Hof E, Ramon H, Swennen RL, Keulemans J (2009) Application of visible and near infrared reflectance spectroscopy (Vis/NIRS) to determine carotenoid contents in banana (Musa spp.) fruit pulp. J Agric Food Chem 57: 17421751

23. Schulz H, Drews H, Quilitzsch R, Krüger H (1998) Application of near infrared spectroscopy for the quantification of quality parameters in selected vegetables and essential oil plants. J Near Infrared Spec. 6A: 125-130

24. Bonierbale M, Gruneberg W, Amoros W, Burgos G, Salas E, Porras E (2009) Total and individual carotenoid profiles in Solanum phureja cultivated potatoes: II. Development of application of near-infrared reflectance spectroscopy (NIRS) calibrations for germplasm characterization. J Food Comp Anal 22: 509-516

25. Clement A, Dorais M, Vernon M (2008) Nondestructive measurement of fresh tomato lycopene content and other physicochemical characteristics using visible-NIRS spectroscopy. J Agric Food Chem 56: 9813-9818

26. Jiménez A (2003) Monitoring carotenoid and chlorophyll pigments in virgin olive oil by visible-near infrared transmittance spectroscopy. On-line application. J Near Infrared Spec 11:219-226 
27. Tkachuk R, Mellish VJ, Daun JK, Macri LJ (1998) Determination of chlorophyll in ground rapeseed using a modified near infrared reflectance spectrophotometer. JAOCS 65: $381-385$

28. Conte LS, Brussolo G, Pizzale L, Carazzolo A, Meurens M, Pavan O (2003) Application of near infrared reflectance analysis to olive oil production quality control. Riv Ital Sostanze Gr 80: 213-217

29. Mailer RJ (2004) Rapid evaluation of olive oil quality by NIR reflectance spectroscopy. JAOCS 8: 823-827

30. Armenta S, Garrigues S, De La Guardia M (2007) Determination of edible oil parameters by near infrared spectrometry. Anal Chim Acta 596: 330-337

31. Bendini A, Cerretani L, Di Virgilio F, Belloni P, Lercker G, Gallina T (2007) Inprocess monitoring in industrial olive mill by means of FT-NIR. Europ J Lipid Sci Technol 109: 498-504

32. Costa AF, Coelho MJ, Gambarra FF, Bezerra SR, Harrop RK, Ugulino MC (2008) NIR spectrometric determination. Food Res Int 41: 341-348

33. Pizarro C, Rodríguez-Tecedor S, Pérez-del-Notario N, Esteban-Díez I, González-Sáiz JM (2013) Classification of Spanish extra virgin olive oils by data fusion of visible spectroscopic fingerprints and chemical descriptors. Food Chem 138: 915-922

34. Tobias RD (1995) An Introduction to Partial Least Squares Regression. SUGI

Proceedings, $20^{\text {th }}$ Annual Conference Pgs. 1250-1257.

Formatted: Superscript 
35. Beebe KR, Kowalski BR. (1987) An introduction to multivariate calibration and analysis. Anal. Chem. 59: 1007A-1017A.

36. Wold S, Sjöström M, Eriksson L (2001) PLS-regression: a basic tool of chemometrics.

\section{Chemometr Intell Lab 58: 109-130}

37. Esbensen KH. Multivariate data analysis - In practice: An introduction to multivariate data analysis and experimental design / Kim H. Esbensen. 5th Ed. Woodbridge, USA. Camo, 2006.

34.38. Wülfert F, Kok WT, Smilde AK (1998) Influence of temperature on vibrational spectra and consequences for the predictive ability of multivariate models. Anal Chem 70: $1761-1767$

35. Wold S, Sjöström M, Eriksson L (2001) PLS-regression: a basic tool of chemometrics. Chemometr Intell Lab 58: 109-130

36.39. Williams PC, Sobering D (1996) How do we do it: A brief summary of the methods we use in developing near infrared calibrations. In Near Infrared Spectroscopy: The future waves, Davies AMC, Williams PC Eds. pp 185-188. NIR Publications, Chichester

\section{Figure captions}

Figure 1. Example of olive oil visible spectrum from Picual (P) Manzanilla (M) and Arbequina (A).

Figure 2. Predictive model VIS for total chlorophylls $\left(\mathrm{Ch}_{1}\right)$ and external validation $\left(\mathrm{Vch}_{1}\right)$. Figure 3. Predictive model VIS for total carotenoids $\left(\mathrm{Ca}_{1}\right)$ and external validation $\left(\mathrm{Vca}_{1}\right)$.
Formatted: Justified, Line spacing: Double

Formatted: Font: $12 \mathrm{pt}$

Formatted: English (U.S.) 
1

2

3

4

5

6

7

8

9

10

11

12

13

14

15

16

17

18

19

20

21

22

23

24

25

26

27

28

29

30

31

32

33

34

35

36

37

38

39

40

41

42

43

44

45

46

47

48

49

50

51

52

53

54

55

56

57

58

59

60

509 Figure 4. Predictive model VIS/NIRS for total carotenoids $\left(\mathrm{Ca}_{4}\right)$ and external validation $510 \quad\left(\mathrm{Vca}_{4}\right)$. 


\begin{tabular}{ccccc}
\hline & \multicolumn{2}{c}{ Total chlorophylls } & \multicolumn{2}{c}{ Total carotenoids } \\
\hline & Calibration & Validation & Calibration & Validation \\
$\mathrm{N}^{\mathrm{a}}$ & 205 & 53 & 205 & 50 \\
Range & $1.39-88.13$ & $1.47-74.35$ & $2.05-38.53$ & $2.62-36.16$ \\
$\bar{X}^{\mathrm{b}}$ & 17.23 & 15.58 & 11.08 & 10.39 \\
$\sigma^{\mathrm{c}}$ & 15.83 & 13.99 & 7.46 & 6.99 \\
\hline
\end{tabular}

Table 1. Statistics of chlorophylls and carotenoids from the calibration and validation sets. ${ }^{\mathrm{a}} \mathrm{N}$, size; ${ }^{\mathrm{b}} \bar{X}$, mean $\left(\mathrm{mg} \mathrm{Kg}^{-1}\right) ;{ }^{\mathrm{c}} \sigma$, standard deviation $\left(\mathrm{mg} \mathrm{Kg}^{-1}\right)$. 


\begin{tabular}{|c|c|c|c|c|c|c|c|c|c|}
\hline \multirow[b]{3}{*}{ Model } & \multicolumn{4}{|c|}{ Total chlorophylls } & \multicolumn{5}{|c|}{ Total carotenoids } \\
\hline & \multicolumn{2}{|c|}{ Calibration } & \multicolumn{2}{|c|}{ Validation } & \multirow[b]{2}{*}{ Model } & \multicolumn{2}{|c|}{ Calibration } & \multicolumn{2}{|c|}{ Validation } \\
\hline & $\mathrm{R}^{2}$ & SEC & SEP & RPD & & $\mathrm{R}^{2}$ & SEC & SEP & RPD \\
\hline $\mathrm{Ch}_{1}-\mathrm{MLR}$ & 0.97 & 2.63 & 2.43 & 5.76 & $\mathrm{Ca}_{1}-\mathrm{PLS}$ & 0.91 & 2.28 & 1.90 & 3.68 \\
\hline $\mathrm{Ch}_{2}-\mathrm{PLS}$ & 0.96 & 3.20 & 2.73 & 5.12 & $\mathrm{Ca}_{2}-\mathrm{PCR}$ & 0.90 & 2.30 & 1.90 & 3.68 \\
\hline $\mathrm{Ch}_{3}-\mathrm{PCR}$ & 0.96 & 3.22 & 2.72 & 5.14 & $\mathrm{Ca}_{3}$ MLR & 0.91 & 2.19 & 2.11 & 3.31 \\
\hline $\mathrm{Ch}_{4}-\mathrm{PLS}^{a}$ & 0.96 & 3.13 & 3.51 & 4.05 & $\mathrm{Ca}_{4}-\mathrm{PLS}^{(l)}$ & 0.95 & 1.74 & 1.81 & 3.86 \\
\hline $\mathrm{Ch}_{5}-\mathrm{SLR}$ & 0.87 & 5.71 & 4.27 & 3.28 & $\mathrm{Ca}_{5}-\mathrm{SLR}$ & 0.81 & 3.27 & 2.63 & 2.66 \\
\hline $\mathrm{Ch}_{6}-\mathrm{PLS}^{b}$ & 0.56 & 10.47 & & & $\mathrm{Ca}_{6}-\mathrm{PLS}^{(2)}$ & 0.62 & 4.58 & & \\
\hline
\end{tabular}

Table 2. Statistics of the models built by Multiple Linear Regression (MLR), Principal Component Regression (PCR), Partial Least Squares (PLS), Simple Linear Regression (SLR), PLS from UV, VIS and NIR regions $\left(\mathrm{PLS}^{a}\right.$ ) and PLS from exclusively NIR region $\left(\mathrm{PLS}^{b}\right) . \mathrm{R}^{2}$, model squared coefficient of calibration; SEC, standard error of calibration; SEP, standard error of performance; RPD, residual predictive deviation. 


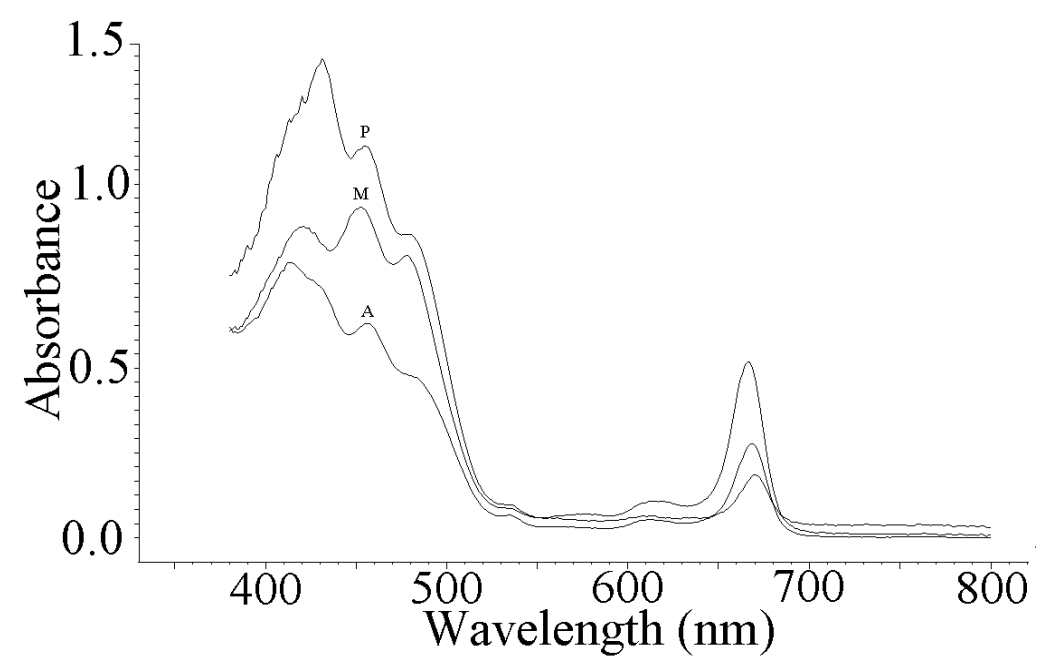

Figure 1 

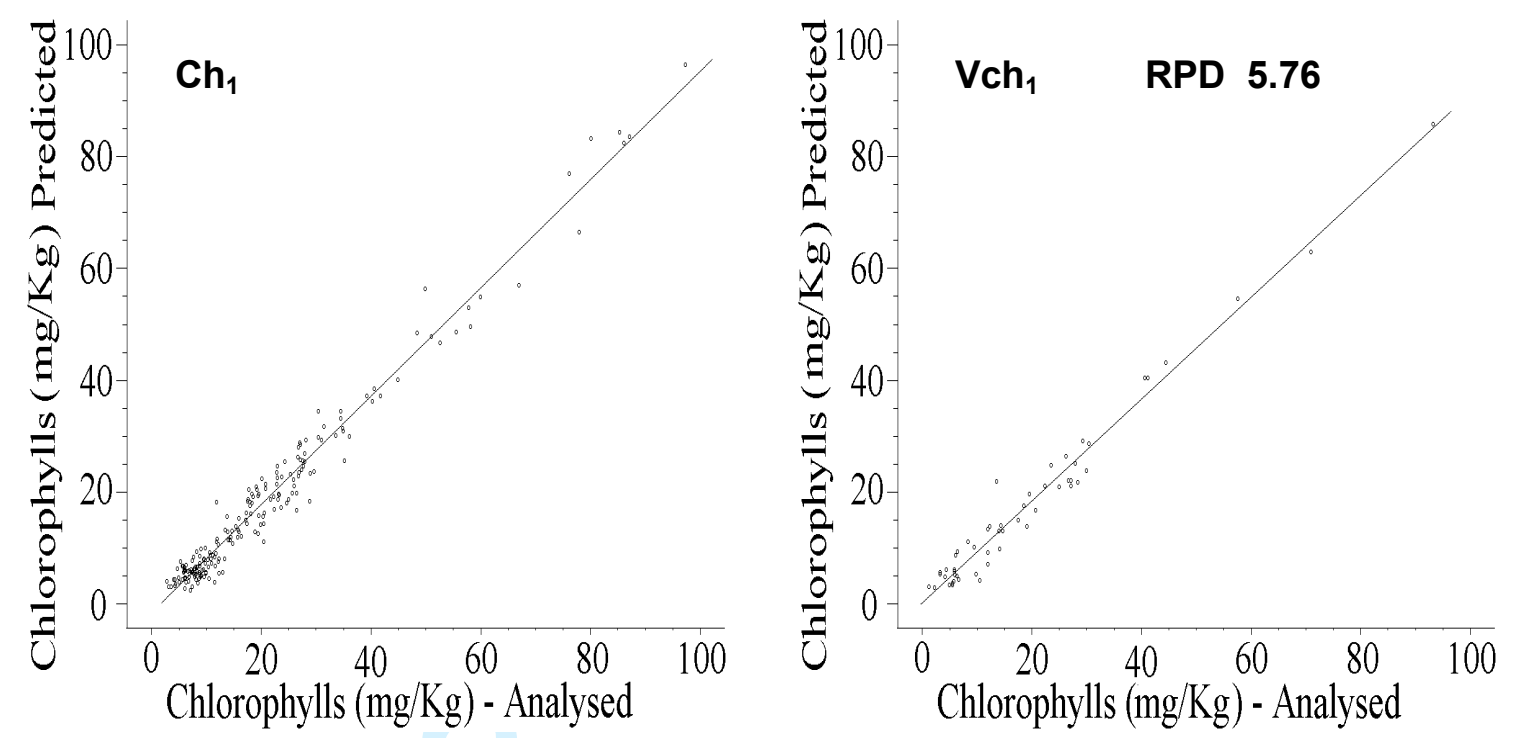

Figure 2. Predictive model VIS for total chlorophylls $\left(\mathrm{Ch}_{1}\right)$ and external validation $\left(\mathrm{Vch}_{1}\right)$. 

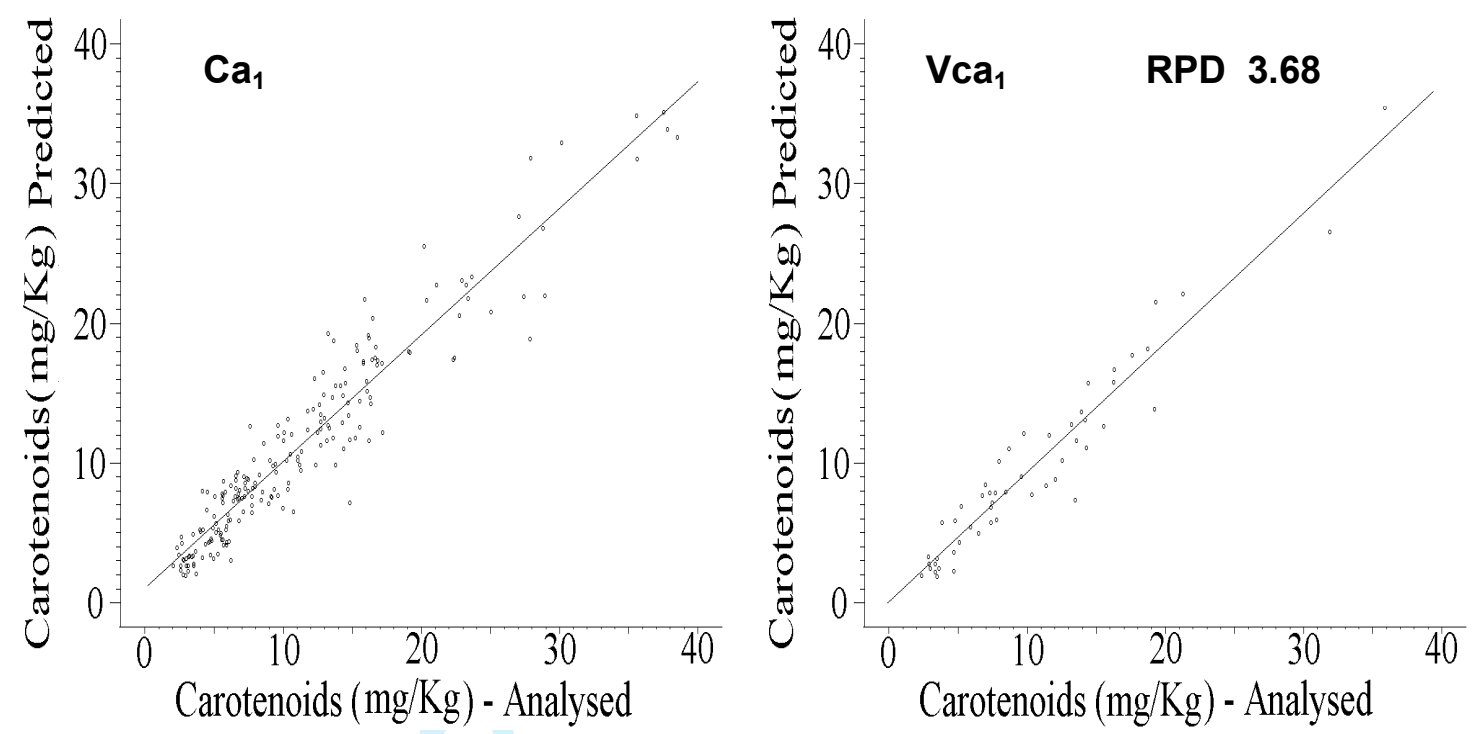

Figure 3 
Page 31 of 31

Journal of the American Oil Chemists

1
2
3
4
5
6
7
8
9
10
11
12
13
14
15
16
17
18
19
20
21
22
23
24
25
26
27
28
29
30
31
32
33
34
35
36
37
38
39
40
41
42
43
44
45
46
47
48
49
50
51
52
53
54
55
56
57
58
59
60
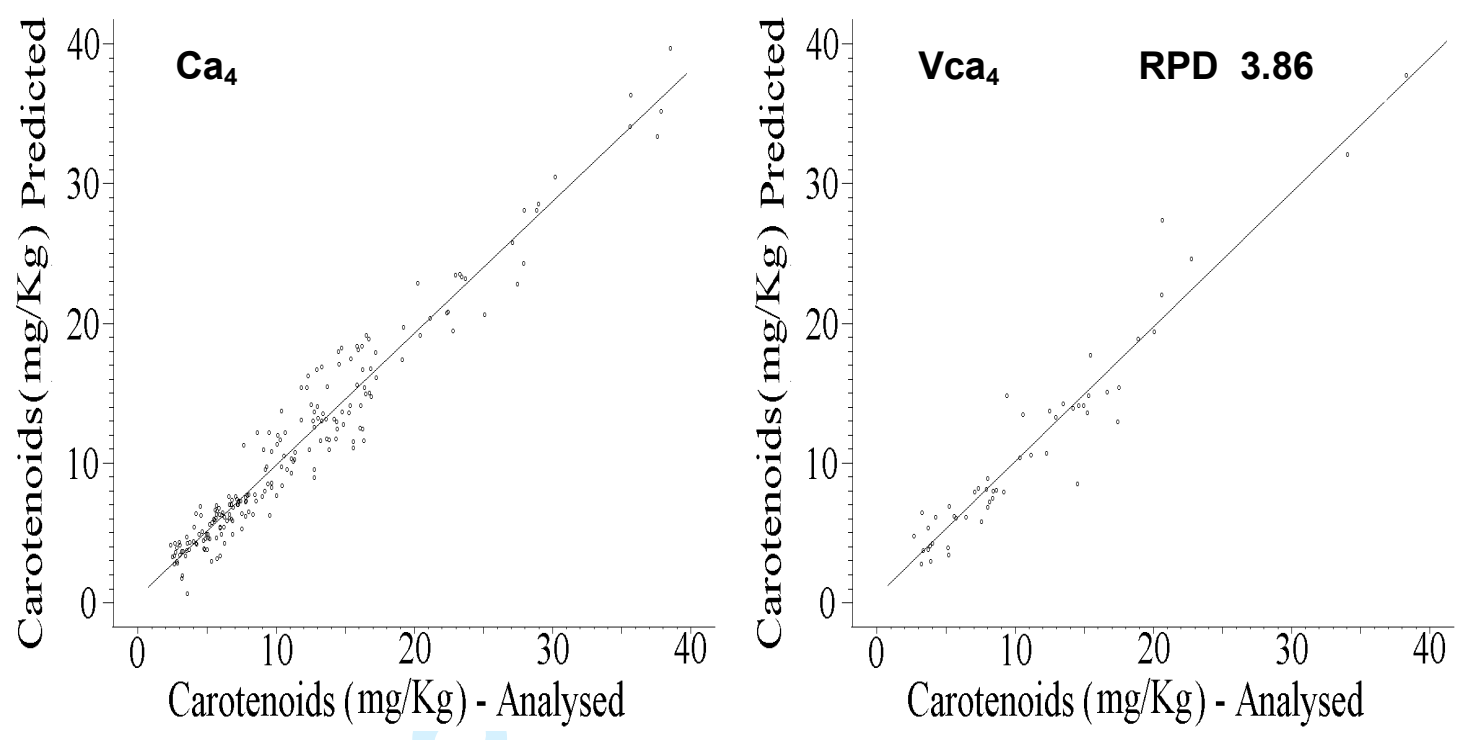

1 Figure 4

3 